\title{
利用稳定氮和碳同位素分析渤海湾食物网 主要生物种的营养层次
}

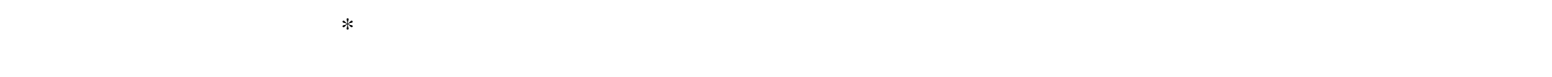
(1)北京大学环境学院, 北京 100871; (2)中国科学院生态环境研究中心环境水质学国家重点实验室, 北京 100085;

(3)日本国土环境株式会社, 东京 154-8585, 日本.*联系人, E-mail: hujy@urban.pku.edu.cn)

摘要 本研究利用稳定氮和碳同位素比值构建了渤海湾食物网主要生物种的营养层次. 其中, $\delta^{13} \mathrm{C}$ 值 范围为-25.38\% - 11.08\%, 并且在水生生物体内没有稳定的富集现象, 梭鱼的生活习性(洄游经济鱼种) 和食性可能导致其 $\delta^{13} \mathrm{C}$ 值明显高于其他鱼种. $\delta^{15} \mathrm{~N}$ 值范围为 4.08\% $~ 13.98 \%$, 随营养层次升高有明显富 集趋势, 富集因子为 $3.8 \%$. 利用 $\delta^{15} \mathrm{~N}$ 建立了稳定同位素比值与营养层次的关系模型, 预测出浮游动植 物、无脊椎生物、鱼类和海鸟的营养层次分别为 1.46 2.10, 1.91 3.32, 2.55 4.23 和 2.98 4.28.

关键词 食物网 营养层次 $\delta^{15} \mathrm{~N} \quad \delta^{13} \mathrm{C} \quad$ 渤海湾

随着对食物网的深入研究, 人们认识到确定生 物种间的食物关系是建立复杂食物网的主要途径, 更能有效地对生态资源进行评估和持续利用. 用来 定量描述食物网中生物种营养层次的传统方法主要 是胃容量分析方法. 该方法直接分析生物体胃中残 留物的组成来计算生物体的营养层次 度上它反映了生物体在采样时期的食性. 但是, 由于 捕食者的食物种类分散于不同营养层次, 并随季节 和地域发生变化, 所以需要在采样区域进行长年采 样分析才能建立准确的营养层次关系, 这就受到了 时间和空间的限制 ${ }^{[3,4]}$. 另外, 该方法还存在对于分 析浮游动植物、底栖生物和其他小型生物的可操作性 差的缺点.

国外的许多研究已经证明, 利用生物体体内的 稳定碳和氮同位素比值能准确定量描述食物网中主 要物质(碳源)的来源和生物种的营养层次 ${ }^{[5,6]}$. 因为 该方法是基于稳定同位素反映了生物体对食物的吸 收并且是生物体长时期代谢的结果这一事实, 这样 既表达了生物体摄取的食物种类信息, 也表达了一 段时间内生物体对食物的吸收信息 ${ }^{[4]}$. 用来分析食物 网生物种间食物关系的稳定同位素有氮、碳和硫等, 其中氮和碳同位素最为常用. 稳定氮同位素比值由 于富集因子较高( $3 \%$ \% $5 \%$ ) 主要用来确定生物种在食

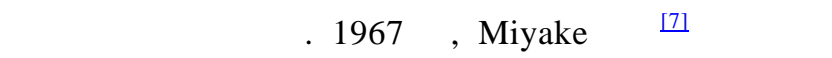
现 $\delta^{15} \mathrm{~N}$ 在食物网中随营养层次升高而增大的现象. 1981 年, Deniro等人 ${ }^{[8]}$ 在室内培养实验中证实这一结 果. 1994 年, Cabana等人 ${ }^{[9]}$ 利用稳定氮同位素比值作
为连续定量描述营养层次的综合指标研究了在加拿 大 24 个湖泊中水银的生物放大现象. 近年来, 稳定 氮同位素比值成为研究污染物在食物网生物富集和 放大效应的重要参数, 开始被应用于水生生态系统 污染物的环境行为研究中 $[4,10,11]$. 稳定碳同位素比值 也常用于生态系统食物链结构的分析 ${ }^{[12,13]}$, 但该比 值随营养层次的富集不明显(富集因子约为 $1 \%$ ), 所 以稳定碳同位素比值主要用来确定生物种的食物组 成以及追踪生态系统中的主要物质(碳源)的来源 [14 16]

渤海是中国重要的内海, 研究者已经对渤海食 物网的种间食物关系进行了大量的研究. 但是, 以往 的研究局限于利用传统的胃容量方法测定主要鱼种 的营养层次 $[1,2,17]$, 至今还没有包括低等生物如浮游 动植物和高等生物如鸟类的食物网各营养级生物的 种间关系的研究. 本研究在渤海湾中采集了浮游动 植物、5 种无脊椎生物、8 种鱼类、海鸟和鸟蛋, 测 定了所有样品的稳定氮和碳同位素比值, 并通过稳 定同位素比值分析了种间食物关系, 建立了稳定氮 同位素比值和营养层次的关系模型, 为研究有毒有 害污染物质(包括持久性有机污染物、内分泌干扰物 质等)在渤海湾食物链中的生物富集提供了基础数据.

\section{1 样品采集与分析方法}

\section{1 样品采集}

本工作选取了渤海湾中常见的经济鱼类、无脊椎 动物、浮游植物。浮游动物、海鸥和鸟蛋. 根据前人 
的研究 ${ }^{[1,2,17]}$, 得出了所选取的水生生物种间食物关 系结构图(图 1). 其中, 海鲶、绵鳚、狼鱼、黄姑鱼、 鲈鱼和鲬为肉食性的, 以虾和幼鱼为主要饵料; 鳀鱼 为杂食性的, 以浮游动物为主要饵料, 也吃虾和其他 鱼类的小鱼; 梭鱼为植食性的, 以浮游动物为主要饵 料. 扇贝、蟹、虾、蛤和螺都属于无脊椎生物, 主要 以浮游动物和底栖生物为食 ${ }^{[1,2,18]}$.

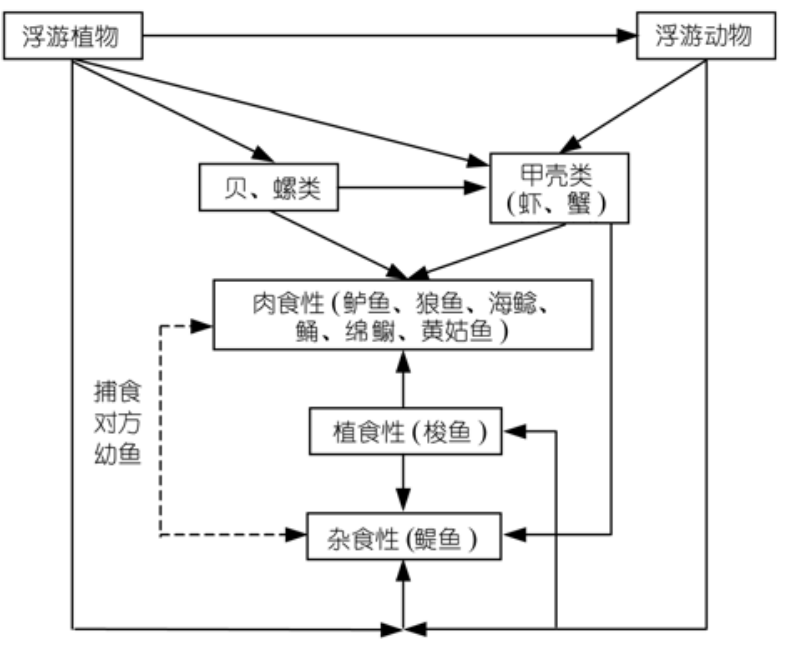

图 1 渤海湾采集生物种的食物关系

天津地区本地留鸟主要是麻雀, 其他留鸟因为 数量稀少成为国家保护动物, 如纵纹腹小鸮和红隼 等. 本研究中, 我们选取了在天津滞留时间长而且种 群数量相对较大的鸥类和行鸟类作为研究对象, 采样 时期选取在海鸥已迁徙至天津一段时间后, 这样海 鸥的食物组成反映了其在当地的食性.

样品采集于 2002 2003 年期间. 采集时间、地点 和对象见表 1 . 其中, 浮游植物和浮游动物的采集采 用国家标准方法(GB 17378): 浮游植物采集网直径为 $37 \mathrm{~cm}$, 长度为 $140 \mathrm{~m}$ (网目: $77 \mu \mathrm{m}$ ); 浮游动物采集网 直径为 $31.6 \mathrm{~cm}$, 长度为 $140 \mathrm{~m}$ (网目: $160 \mu \mathrm{m})$, 样品 来自渤海湾的 6 个采样点 $\left(39^{\circ} 00^{\prime} \mathrm{N}, 117^{\circ} 53^{\prime} \mathrm{E}\right.$; $39^{\circ} 00^{\prime}$ $\mathrm{N}, 118^{\circ} 00^{\prime} \mathrm{E} ; 38^{\circ} 45^{\prime} \mathrm{N}, 117^{\circ} 53^{\prime} \mathrm{E} ; 38^{\circ} 45^{\prime} \mathrm{N}, 118^{\circ} 00^{\prime} \mathrm{E}$; $38^{\circ} 30^{\prime} \mathrm{N}, 117^{\circ} 53^{\prime} \mathrm{E}$ 和 $\left.38^{\circ} 30^{\prime} \mathrm{N}, 118^{\circ} 00^{\prime} \mathrm{E}\right)$. 调查发现
浮游植物的优势种是硅藻门(Bacillariophyta)和甲藻 门(Pyrrophyta), 浮游动物的优势种为桡足类中的长 腹剑水蚤 (Oithona similis), 双刺纺锤水蚤 (Acartia bifilosa), 小拟哲水蚤(Paracalanus parvus)和真刺唇 角水蚤 (Labidocera euchaeta), 这与渤海近海调查结 果相似 ${ }^{[2]}$. 虾蟹等无脊椎动物和鱼类用渔网采集; 海鸥由捕鸟夹采集. 采来的样品冷冻保存在 $-20^{\circ} \mathrm{C}$ 冰 箱中.

\section{2 碳、氮稳定同位素分析方法}

鱼类和鸟类以肌肉作样品, 浮游植物、浮游动物 和无脊椎动物取生物个体的全体, 所有样品首先被 匀浆机磨成均质浆状, 然后经过冷冻干燥得到干粉 状样品. 称取约 $100 \mathrm{mg}$ 生物样品, 加入约 $10 \mathrm{ml}$ 甲醇 振荡并静置一晚用于去除生物样品中的脂肪(脂肪会 影响碳同位素丰度的测定 ${ }^{[6]}$ ). 受甲醇浸泡过的样品 再用约 $10 \mathrm{ml}$ 甲醇洗一次, 并在 $80^{\circ} \mathrm{C}$ 下烘干且稳定 $4 \mathrm{~h}$. 准确称取 $0.5 \mathrm{mg}$ 处理好的样品, 将其装在锡制的胶 囊中, 并将胶囊压实成锡球(其间不留空气). 锡球即 可直接利用同位素分析仪进行分析测定. 仪器首先 将锡球在 $1000 \sim 1050^{\circ} \mathrm{C}$ 下灼烧, 灼烧产物经载气(氦 气)通过 $\mathrm{Cr}_{2} \mathrm{O}_{3}$ 和活性 $\mathrm{Cu}$ 柱净化. 这样, 生物样品中的 碳和氮可完全转化为 $\mathrm{N}_{2}$ 和 $\mathrm{CO}_{2}$ 气体, 经过 $\mathrm{Mg}\left(\mathrm{ClO}_{4}\right)_{2}$ 脱水, 并用气相色谱柱(Porapak QS)分离后, 进行质 谱分析(THERMO Delta plus, Finnigan MAT). 仪器分 析可以测出生物样品中 ${ }^{15} \mathrm{~N} /{ }^{14} \mathrm{~N}$ 和 ${ }^{13} \mathrm{C} /{ }^{12} \mathrm{C}$ 的比值. $\delta^{15} \mathrm{~N}$ 和 $\delta^{13} \mathrm{C}$ 按以下公式计算得出:

$$
\begin{aligned}
\delta^{15} \mathrm{~N} & =\left(\frac{{ }^{15} \mathrm{~N} /{ }^{14} \mathrm{~N}_{\text {sample }}}{{ }^{15} \mathrm{~N} /{ }^{14} \mathrm{~N}_{\text {atmosphere }}}-1\right) \times 1000, \\
\delta^{13} \mathrm{C} & =\left(\frac{{ }^{13} \mathrm{C} /{ }^{12} \mathrm{~N}_{\text {sample }}}{{ }^{13} \mathrm{C} /{ }^{12} \mathrm{C}_{\mathrm{PDB}}}-1\right) \times 1000,
\end{aligned}
$$

式中, ${ }^{15} \mathrm{~N} /{ }^{14} \mathrm{~N}_{\text {atmosphere }}$ 为标准大气氮同位素比值; ${ }^{13} \mathrm{C} /{ }^{12} \mathrm{C}_{\mathrm{PDB}}$ 为国际标准物质 $\mathrm{PDB}$ (Peedee Belemnite carbonate)的碳同位素比值.

\begin{tabular}{|c|c|c|c|c|c|}
\hline 时间 & 2002 年 6 月 & 2002 年 8 月底 & 2002 年 11 月底 & 2003 年 7 月底 & 2003 年 6 月中 \\
\hline & (1)天津汉沽采集鱼和无脊椎 & (1)天津汉沽采集鱼和无脊椎 & & & \\
\hline 采样地点和 & 动物 & 动物 & 天津蓟运河河口采 & 天津汉沽采集鱼和 & 天津大港水库芦苇 \\
\hline 对象 & $\begin{array}{c}\text { (2)渤海湾中部采集浮游植物和 } \\
\text { 浮游动物 }\end{array}$ & $\begin{array}{c}\text { (2)渤海湾中部采集浮游植物和 } \\
\text { 浮游动物 }\end{array}$ & 集海鸥 & 无脊椎动物 & 丛中采集鸟蛋 \\
\hline
\end{tabular}

表 1 生物样品采集时间和地点 
表 2 渤海湾采集生物种的样品数 $n, \delta^{15} \mathrm{~N}, \delta^{13} \mathrm{C}$ 以及计算出的营养层次(Mean $\pm \mathrm{SD}$ )

\begin{tabular}{|c|c|c|c|c|}
\hline 种类 & 营养层次 & $\delta^{13} \mathrm{C}$ & $\delta^{15} \mathrm{~N}$ & $n$ \\
\hline 浮游植物(Phytoplankton) & $1.61 \pm 0.14$ & $-20.28 \pm 1.33$ & $4.67 \pm 0.54$ & $3^{\text {a) }}$ \\
\hline 浮游动物(Zooplankton) & $2.00 \pm 0.14$ & $-19.42 \pm 1.07$ & $6.14 \pm 0.51$ & $3^{\mathrm{a})}$ \\
\hline 扇贝(Argopecten irradians) & $2.15 \pm 0.12$ & $-18.00 \pm 0.79$ & $6.71 \pm 0.79$ & 3 \\
\hline 蟹(Portunus trituberculatus) & $3.10 \pm 0.17$ & $-15.83 \pm 0.89$ & $10.33 \pm 0.64$ & 3 \\
\hline 虾(upogebia sp.) & $3.16 \pm 0.14$ & $-14.99 \pm 0.36$ & $10.55 \pm 0.54$ & 3 \\
\hline 蛤(Ruditapes philippinarum) & $2.17 \pm 0.20$ & $-15.71 \pm 0.10$ & $6.78 \pm 0.75$ & 3 \\
\hline 螺(Rapana venosa) & $2.79 \pm 0.12$ & $-16.97 \pm 0.50$ & $9.14 \pm 0.44$ & 3 \\
\hline 海鲶(Chaeturichthys sitgmatias) & $3.67 \pm 0.04$ & $-15.36 \pm 0.51$ & $12.48 \pm 0.14$ & 3 \\
\hline 鲈鱼(Lateolabras japonicus) & $3.88 \pm 0.49$ & $-14.28 \pm 2.07$ & $13.28 \pm 1.87$ & 2 \\
\hline 鲬(Platycephalus indlcus) & $3.65 \pm 0.24$ & $-17.20 \pm 0.86$ & $12.41 \pm 0.90$ & 3 \\
\hline 狼鱼(Obontamblyopus rubicundus) & $3.58 \pm 0.11$ & $-13.99 \pm 1.26$ & $12.13 \pm 0.43$ & 3 \\
\hline 梭鱼(Liza so-iuy) & $3.01 \pm 0.44$ & $-11.59 \pm 0.82$ & $9.97 \pm 1.68$ & 3 \\
\hline 绵鳚(Enchelyopus elongatus) & $3.87 \pm 0.07$ & $-13.07 \pm 0.84$ & $13.23 \pm 0.26$ & 3 \\
\hline 黄姑鱼(Nibea albiflora) & $3.65 \pm 0.39$ & $-17.60 \pm 1.35$ & $12.39 \pm 1.48$ & 3 \\
\hline 鳀鱼(Engraulis japonicus) & $3.65 \pm 0.09$ & $-17.99 \pm 0.08$ & $12.41 \pm 0.35$ & 3 \\
\hline 银鸥(Larus argentatus) & $3.84 \pm 0.43$ & $-22.19 \pm 1.86$ & $11.74 \pm 1.63$ & 6 \\
\hline 蛋(红嘴鸥 Larus ridibundus Linne) & $3.04 \pm 0.08$ & $-20.86 \pm 2.16$ & $8.77 \pm 0.27$ & 3 \\
\hline 蛋(须浮鸥 Chlidonias hybrida swinhoei) & $3.19 \pm 0.11$ & $-22.27 \pm 2.08$ & $9.42 \pm 0.41$ & 2 \\
\hline
\end{tabular}

a) 为来自 6 个采样点的 3 个样品

\section{2 结果和讨论}

\section{$2.1 \delta^{15} \mathrm{~N}$ 和 $\delta^{13} \mathrm{C}$ 测定结果}

表 2 表示了实验测定的 $\delta^{15} \mathrm{~N}$ 和 ${ }^{13} \mathrm{C}$ 值. 可以看 出, $\delta^{15} \mathrm{~N}$ 和 $\delta^{13} \mathrm{C}$ 数值跨度很大, $\delta^{13} \mathrm{C}$ 从银鸥肌肉的 $-25.38 \%$ o 到梭鱼的 $-11.08 \%, \delta{ }^{15} \mathrm{~N}$ 从浮游植物的 $4.08 \%$ 到黄姑鱼的 $13.98 \%$, 并且水生生物的 $\delta^{15} \mathrm{~N}$ 随 $\delta^{13} \mathrm{C}$ 增大而增大.

尽管一些海洋生态系统或单一食物关系的研究 表明 $\delta^{13} \mathrm{C}$ 值随营养层次的富集因子约为 $1 \%{ }^{[14,16,19]}$, 我们的结果并没有发现稳定的 $\delta^{13} \mathrm{C}$ 值富集现象. 这 与Hobson等人 ${ }^{[6]}$ 在北冰洋和Wada等人 ${ }^{[20]}$ 在南冰洋海 洋生态系统的发现一致. Wada等人 ${ }^{[20]}$ 指出 $\delta^{13} \mathrm{C}$ 值不 富集是由于样品的脂肪造成, 但Hobson等人 ${ }^{[6]}$ 对已 去除脂肪的样品分析得出的结论是脂肪不是导致 $\delta$ ${ }^{13} \mathrm{C}$ 值不富集的直接原因, 这与我们的结果一致. 浮 游动物和浮游植物 ${ }^{13} \mathrm{C}$ 值的富集值为 $1 \%$, 这与蔡德 陵等人 12$]$ 得出的渤海浮游生物群体富集因子 (1.7\%) 相似. 散贝、虾、蟹、螺和蛤与浮游动植物的富集值 很大 $\left(2.28 \%\right.$ 4.57\%o), 这与McConnaughey等人 ${ }^{[21]}$ 在 白令海和Hobson等人 ${ }^{[6]}$ 在北冰洋发现的双壳类无脊 椎生物的高 $\delta{ }^{13} \mathrm{C}$ 值富集相似. McConnaughey等人 ${ }^{[21]}$ 把原因归结为底栖生物具有强碳同位素富集能力, 或者在底栖生物和浮游动植物两级营养层之间还存 在细菌或较小型底栖生物作为中间层. 从图 2 中可以 看出, 海鲶、鲇鱼、狼鱼和绵鳚的 $\delta^{13} \mathrm{C}$ 值接近, 鲬、 黄姑鱼和鳀鱼的 $\delta{ }^{13} \mathrm{C}$ 值接近, 表明其食物组成和生

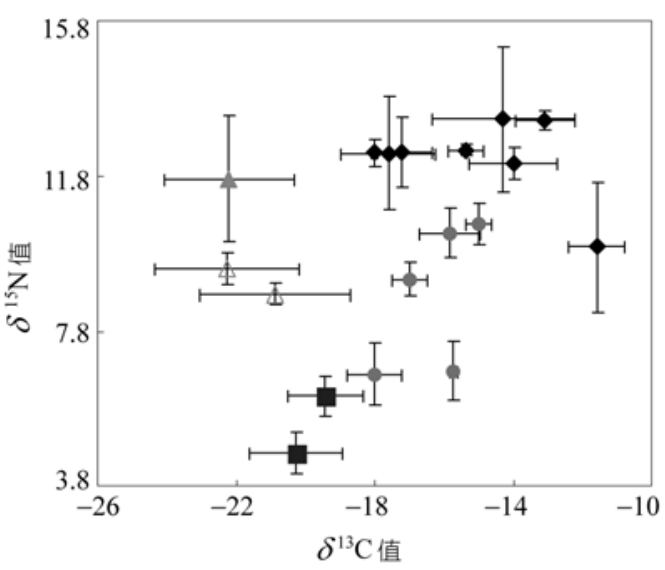

图 2 渤海湾食物网各生物体 $\delta^{15} \mathrm{~N}$ 和 $\delta^{13} \mathrm{C}$ 值 $\Delta$ 示银鸥; $\triangle$ 示鸟蛋; 示初级生产者和消费者

活习性可能比较相似. 值得注意的是梭鱼的 ${ }^{13} \mathrm{C}$ 值 明显高于其他鱼种, 这可能与梭鱼的生活习性(洄游 经济鱼种)和食性有关.

与 $\delta^{13} \mathrm{C}$ 相比, $\delta^{15} \mathrm{~N}$ 在食物网中富集更为明显. 比 如, 浮游动物与浮游植物的 $\delta^{15} \mathrm{~N}$ 差值为 $1.47 \%$, 甲壳 类生物与浮游植物的 $\delta^{15} \mathrm{~N}$ 差值为 $2.04 \% \sim 4.47 \%$, 虾 和蟹与浮游动物的 $\delta^{15} \mathrm{~N}$ 差值为 $4.18 \%$ 4.40\%, 梭鱼 主要以浮游动物为食 $(3.82 \%)$ 是最基础的鱼类, 其他 鱼类基本处于同一营养层次, 它们与梭鱼 $\delta^{15} \mathrm{~N}$ 差值 在 $2.16 \%$ 3.31\%o的范围内. 由一些主要摄食关系生 物的平均稳定氮同位素比值差异(如虾蟹与浮游动物, 梭鱼与浮游动物等) 可以获得渤海湾水生食物网稳定 
氮同位素的富集因子为 $3.8 \%$. 这与Hobson等人 ${ }^{[6]}$ 在 北冰洋海洋生态系统和Kidd等人 ${ }^{[4]}$ 在中纬淡水湖生 态系统(3.8\%o), 以及Wada等人 ${ }^{[20]}$ 在南冰洋海洋生态 系统(3.3\%o)得到的富集因子相似.

与水生生物不同, 海鸟属于恒温动物, 主要以鱼 类为食, 处在食物网的最高营养层次上. 分析时, 我 们测定了银欧的肌肉以及红嘴欧和须浮鸥鸟蛋中稳 定碳和氮同位素比值. 与肌肉的同位素比值相比, 蛋 同位素比值反应了乌在孵蛋期间的食物组成 ${ }^{[22]}$. 测 定结果显示, 银鸥的 $\delta{ }^{13} \mathrm{C}$ 值在-25.38\% $~-19.81 \%$ 的 范围内, 红嘴欧和须浮鸥鸟蛋的 $\delta{ }^{13} \mathrm{C}$ 值在 $-23.74 \%$ - 18.37\%o的范围内, 均高于Rau等人 ${ }^{[23]}$ 在威 德尔海调查得到的海鸟体内 $\delta^{13} \mathrm{C}$ 值, 但是低于Jarman 等人 ${ }^{[22]}$ 在Farallones海湾测定的海鸟体内 $\delta{ }^{13} \mathrm{C}$ 值, 说 明不同地域的海鸟受食性和生活习性影响具有不同 的稳定碳同位素比值. 银鸥的稳定氮同位素比值 (9.71\%o 13.42\%o)要高于食物网中其他生物, 说明其 处于食物网的高营养层中. 而红嘴欧和须浮鸥鸟蛋 的稳定氮同位素比值较低, 这可能是因为鸟蛋没有 经过成鸟长期的食物积累过程导致的.

\section{$2.2 \delta^{15} \mathrm{~N}$ 和营养层次的关系模型的建立}

大量的研究和调查表明食物网中稳定氮同位素 比值随营养层次升高会出现稳定富集现象, 因此生 物体在食物网中的营养层次可以用稳定氮同位素比 值的高低表征, 其具体的计算公式如下 ${ }^{[4,10,11]}$ :

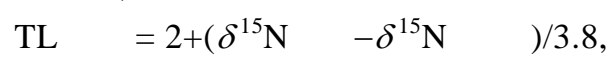

式中, 2 假定为浮游动物营养层次; 3.8 为 $\delta^{15} \mathrm{~N}$ 富集因 子. 由于海鸥与水生生物不同, 属于恒温动物并且排 泄的是尿酸而不是尿素, 所以它与被捕食鱼类的 $\delta^{15} \mathrm{~N}$ 富集值和水生食物网的富集因子不同 ${ }^{[6]}$. Mizutani等人 ${ }^{[24]}$ 经过 23 年观测得出鸟捕食鱼类的 $\delta{ }^{15} \mathrm{~N}$ 富 集因子为 $2.4 \%$. 因此可以得到鸟捕食生物的营养层 次关系公式:

$\mathrm{TL}_{\text {乌 }}=\mathrm{TL}_{\text {水中捕食者 }}+1 ; \delta^{15} \mathrm{~N}$ 乌 $=\delta^{15} \mathrm{~N}_{\text {水中捕食者 }}+2.4$. 所以, 乌的 $\delta^{15} \mathrm{~N}$ 与营养层次关系为:

$\mathrm{TL}_{\text {g }}=3+\left(\delta^{15} \mathrm{~N}_{\text {乌 }}-2.4-\delta^{15} \mathrm{~N}_{\text {浮游动物 }}\right) / 3.8$.

这样, 我们通过测定稳定氮同位素就能准确得 出生物体所在食物网的营养层次(表 2), 同时可以看 出从渤海湾中所捕获生物的食物网的营养层次长度 为 4 级(图 3). 传统胃容量方法在渤海食物网的调查 显示鱼类营养层次分布在 3.0 4.1 之间 ${ }^{[2]}$, 这与本研 究的调查结果类似(鱼类营养层次为 3.01 3.88), 也证

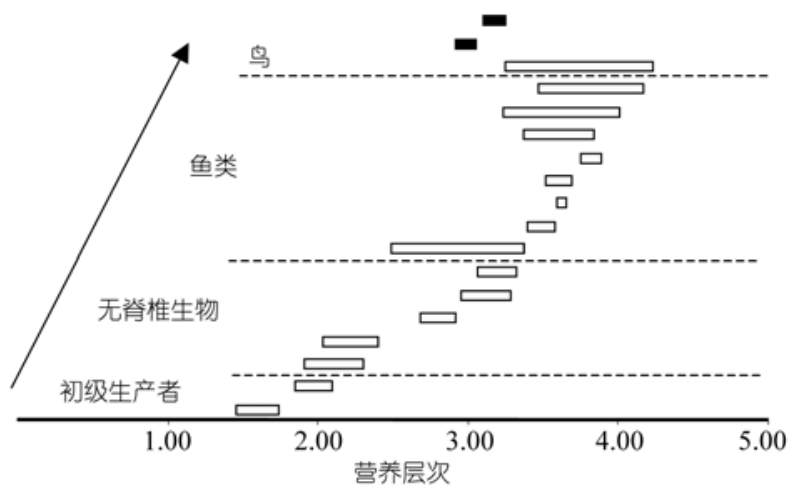

图 3 渤海湾食物网中各生物种的营养层次 口示鸟蛋

明了该测定方法以及建立模型的可靠性.

\section{3 结论}

本研究利用稳定碳氮同位素方法调查了渤海湾 主要生物的种间关系, 其中稳定碳同位素比值直观 地反映了各生物种间的食物关系, 而建立的稳定氮 同位素比值和营养层次的关系模型准确地计算各生 物的营养层次. 与传统的胃容量分析方法相比, 稳定 同位素比值方法更能准确地反映食物网各生物种的 信息. 而且, 连续定量地描述食物网各生物种的营养 层次为以后研究有毒有害污染物质(包括持久性有机 污染物、内分泌干扰物质等)在渤海湾食物链中的生 物富集研究提供了基础数据.

致谢 本研究得到国家重点基础研究发展规划项目(编号: 2003CB415004)、国家自然科学基金优秀创新群体(批准号: 40021101)和日本国际协力机构(JICA)共同资助.

\section{参考文献}

1 邓景耀, 姜卫民, 杨纪明, 等. 渤海主要生物种间关系及食物网 的研究. 中国水产科学, 1997, 4: 1 7

2 苏纪兰, 唐启升. 中国海洋生态系统动力学研究 (II)渤海生态 系统动力学过程. 北京: 科学出版社, 2002. 216 217

3 Paine R T. Food webs: Road maps of interactions or grist for theoretical development? Ecology, 1998, 69: 1648 1654

4 Kidd K A, Schindler D W, Hesslein R H, et al. Correlation between stable nitrogen isotope ratios and concentrations of organochlorines in biota from a freshwater food web. The Science of the Total Environment, 1995, 160/161: 381 390[DOI]

5 Peterson B J. Stable isotopes as tracers of organic matter input and transfer in benthic food webs: A review. Acta Oecologica, 1999, 20(4): 479 487[DOI]

6 Hobson K A, Welch H E. Determination of trophic relationships within a high arctic marine food web using $\delta^{13} \mathrm{C}$ and $\delta^{15} \mathrm{~N}$ analysis. Mar. Ecol. Prog. Ser., 1992, 84: 9 18

7 Miyake Y, Wada E. The abundance ratio of ${ }^{15} \mathrm{~N} /{ }^{14} \mathrm{~N}$ in marine environments. Records of Oceanographic Works in Japan, 1967, 9: /32 5960/04td0283

8 Deniro M J, Epstein S. Influence of diet on the distribution of ni- 
trogen in animals. Geochimica et Cosmochimica Acta, 1981, 45 341 351[DOI]

9 Cabana G, Rasmussen J B. Modelling food chain structure and contaminant bioaccumulation using stable nitrogen isotopes. Nature, 1994, 372: 255 257[DOI]

10 Fisk A T, Hobson K A, Norstrom R J. Influence of chemical and biological factors on trophic transfer of persistent organic pollutants in the northwater Polynya marine food web. Eeviron. Sci. Technol., 2001, 35: 732 738[DOI]

11 Muir D, Savinova T, Savinov V, et al. Bioaccumulation of PCBs and chlorinated pesticides in seals, fishes and invertebrates from the White Sea, Russia. The Science of the Total Environment, 2003, 306: 111 131[DOI]

12 蔡德陵, 王荣, 毕洪生. 渤海生态系统的营养关系: 碳同位素研 究的初步结果. 生态学报, 2001, 21(8): 1354 1359

13 易现峰, 张晓爱, 李来兴, 等. 高寒草甸生态系统食物链结构分 析一一来自稳定性碳同位素的证据. 动物学研究, 2003，25(1): $1 \sim 6$

14 Deniro M J, Epstein S. Influence of diet on the distribution of carbon isotopes in animals. Geochimica et Cosmochimica Acta, 1978, 42: 495 506

15 Fry B, Jeng J W, Scalan R S, et al. ${ }^{13} \mathrm{C}$ food web analysis of a Texas sand dune community. Geochimica et Cosmochimica Acta, 1978, 42: 1299 1302

16 Rau G H, Mearns A J, Young D R, et al. Animal ${ }^{13} \mathrm{C} /{ }^{12} \mathrm{C}$ correlates with trophic level in pelagic food webs. Ecology, 1983, 64:
1314 1318

17 邓景耀, 孟田湘, 任胜民, 等. 渤海鱼类的食物关系. 海洋水产 研究, 1988, (9): 151 172

18 孟庆闻, 廖学祖, 俞泰济, 等. 鱼类学[形态. 分类]. 上海: 上海 科学技术出版社, 1989. 181 205

19 Mills E L, Pittman K, Tan F C. Food-web structure on the Scotian Shelf, eastern Canada: A study using ${ }^{13} \mathrm{C}$ as a food-chain tracer. Rapp. P.-v. Réun. Cons. Int. Explor. Mer, 1984, 183: 111 118

20 Wada E, Terazaki M, Kabaya Y, et al. ${ }^{15} \mathrm{~N}$ and ${ }^{13} \mathrm{C}$ abundances in the Antarctic Ocean with emphasis on the biogeochemical structure of the food web. Deep Sea Res., 1987, 34: 829 841[DOI]

21 McConnaughey T, McRoy C P. Food-web structure and the fractionation of carbon isotopes in the Bering Sea. Mar. Biol., 1979, 53: $257 \sim 262$

22 Jarman W M, Hobson K A, Sydenman W J, et al. Influence of trophic position and feeding location on contaminant levels in the gulf of the Farallones food web revealed by stable isotope analysis. Environ. Sci. Technol., 1996, 30: 654 660[DOI]

23 Rau G H, Ainley D G, Bengtson J L, et al. ${ }^{15} \mathrm{~N} /{ }^{14} \mathrm{~N}$ and ${ }^{13} \mathrm{C} /{ }^{12} \mathrm{C}$ in Weddell Sea birds, seals, and fish: Implications for diet and trophic structure. Mar. Ecol. Prog. Ser., 1992, 84: 1 8

24 Mizutani H, Kabaya Y, Wada E. Nitrogen and carbon isotope compositions relate linearly in cormorant tissues and its diet. Isotopenpraxie, 1991, 4: 166 168

(2004-06-08 收稿, 2005-01-11 收修改稿) 